\title{
PHARMACOKINETIC PROOFS ON INTERACTION BETWEEN ZOLPIDEM AND PHENYTOIN: A TWO-TREATMENT, TWO-PERIOD STUDY IN HEALTHY MALE SUBJECTS
}

\author{
DANA MARIA MUNTEAN ${ }^{a}$, MARIA ADRIANA NEAG ${ }^{b}$, \\ DANIELA PETRUTA PRIMEJDIE ${ }^{*}$, ADINA POPA ${ }^{c}$, \\ MARCELA ACHIMa, LAURIAN VLASE ${ }^{\mathrm{a}}$
}

\begin{abstract}
In clinical practice, the simultaneous use of zolpidem and phenytoin cannot always be avoided, although it can be associated with additive depressants effects on the central nervous system. Considering the common metabolic pathways involving CYP3A and CYP2C, a pharmacokinetic interaction between phenytoin and zolpidem is possible, although not previously quantified. The study was designed as a non-randomized, two-period preclinical trial. Twenty male subjects were included in a study consisting of two periods. Between these, subjects were treated for 6 days with a single daily dose of $150 \mathrm{mg}$ phenytoin. For each treatment period, pharmacokinetic parameters of zolpidem were determined. The multiple-dose administration of phenytoin influenced zolpidem's pharmacokinetics in healthy volunteers, decreasing its exposure through enzymatic induction.
\end{abstract}

Keywords: zolpidem; phenytoin; drug interaction; enzymatic induction

\section{INTRODUCTION}

Insomnia is a prevalent and undertreated sleep disorder, having diverse and interrelated consequences, with Z-drugs among the preferred therapeutic options [1]. Zolpidem (Fig. 1) is indicated for the short-term treatment

\footnotetext{
a "Iuliu Haţieganu" University of Medicine and Pharmacy, Faculty of Pharmacy, Department of Pharmaceutical Technology and Biopharmaceutics, 41 Victor Babes Street, RO-400012, Cluj-Napoca, Romania

b "Iuliu Haţieganu" University of Medicine and Pharmacy, Faculty of Medicine, Department of Pharmacology, Toxicology and Clinical Pharmacology, 41 Victor Babes Street, RO-400012, Cluj-Napoca, Romania

" Iuliu Haţieganu" University of Medicine and Pharmacy, Faculty of Pharmacy, Department of Clinical Pharmacy, 12 Ion Creanga Street, RO-400010, Cluj-Napoca, Romania

* Corresponding author: danaprimejdie@umfcluj.ro
} 
of insomnia, characterized by difficulties with sleep initiation or by difficulty returning to sleep after middle-of-the-night awakening [2]. Available clinical experience confirmed its positive effect on subjective and objective measures of sleep, in both acute and chronic insomnia as well as the absence of rebound insomnia in situations of intermittent administration [3,4]. It is also under investigation for several other indications as the management of restless legs syndrome, various disorders of consciousness or persistent pain $[5,6,7]$.

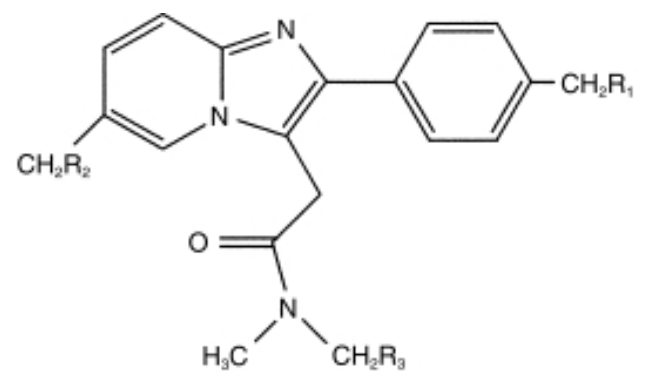

Figure 1. Chemical structure of zolpidem

Zolpidem activates the a1-containing GABAA (BZ1) receptor, with a reduced influence on the $\alpha 2, \alpha 3$ or $\alpha 5$ subunits [8]. Its selectivity explains the reduced myorelaxant or anticonvulsant effects and the preservation of deep sleep (stages 3 and 4) at hypnotic doses [9]. Zolpidem has an approximately $70 \%$ bioavailability. The elimination half-life is about $2.5-2.6$ hours [2]. CYP3A4 is mainly involved in its metabolism (61\% of the net intrinsic clearance), with a more reduced contribution of CYP1A2, 2C9, 2D6, and 2C19, respectively [10].

Given their multiple and complex mechanisms of action, antiepileptic drugs are used for the treatment and prevention of seizures, and for the management of various nonepileptic neurologic or psychiatric disorders [11]. Phenytoin is labeled for the treatment and prophylaxis of seizures during and following neurosurgery or secondary to traumatic brain injuries, as a treatment of generalized tonic-clonic and complex partial seizures and for the management of trigeminal neuralgia [12]. It can also be recommended for the management of the peripheral nervous system disorders secondary to brain tumors or HIV / AIDS infection $[13,14,15]$.

Phenytoin slows the rate of reactivation of voltage-dependent sodium channels after depolarization. The anticonvulsant activity does not cause general depression of the central nervous system and the therapeutic drug levels in the cerebrospinal fluid correlate with the free plasmatic concentration (the anticonvulsant activity is usually obtained at levels of $10-20 \mu \mathrm{g} / \mathrm{mL}$ ) [16]. It presents an oral bioavailability of $20 \%-90 \%$ and it is highly protein-bound (88 - 93\%). It is metabolized by a saturable and genetically polymorphic, hepatic process via the CYP2C9 and CYP2C19 isoenzymes [17]. One population 
pharmacokinetic analysis conducted in various patients groups, suggested a linear two-compartment model for phenytoin [18]. The average half-life of phenytoin is about 24 hours for most patients in the low to mid therapeutic range, showing large age-dependent interindividual variations (12-60 hours) [19]. Pharmacokinetic drug interactions could be related to impaired absorption, to plasmatic protein displacement or to altered metabolism, as phenytoin is a CYP2C9 and CYP2C19 substrate and a strong inducer for CYP3A4, CYP2C9, CYP2C19 and CYP2B6 [20,21], respectively. Therefore, the therapeutic drug monitoring of phenytoin remains a challenging aspect of its therapeutic use, considering its non-linear pharmacokinetics, zero-order elimination and multilevel drug-drug interactions [22].

In clinical practice, the simultaneous use of multiple drugs acting on the central nervous system is frequent, although it is associated with potential additive depressant effects [9]. Moreover, considering the common metabolic pathways involving CYP3A and CYP2C, a pharmacokinetic interaction between phenytoin and zolpidem is possible, although not previously quantified. Therefore, the aim of this study was to evaluate the magnitude of such a pharmacokinetic interaction in healthy volunteers.

\section{RESULTS AND DISCUSSION}

The mean plasma concentrations for zolpidem when administered alone, or in combination with phenytoin after 6 days of treatment with phenytoin are shown in Figure 2.

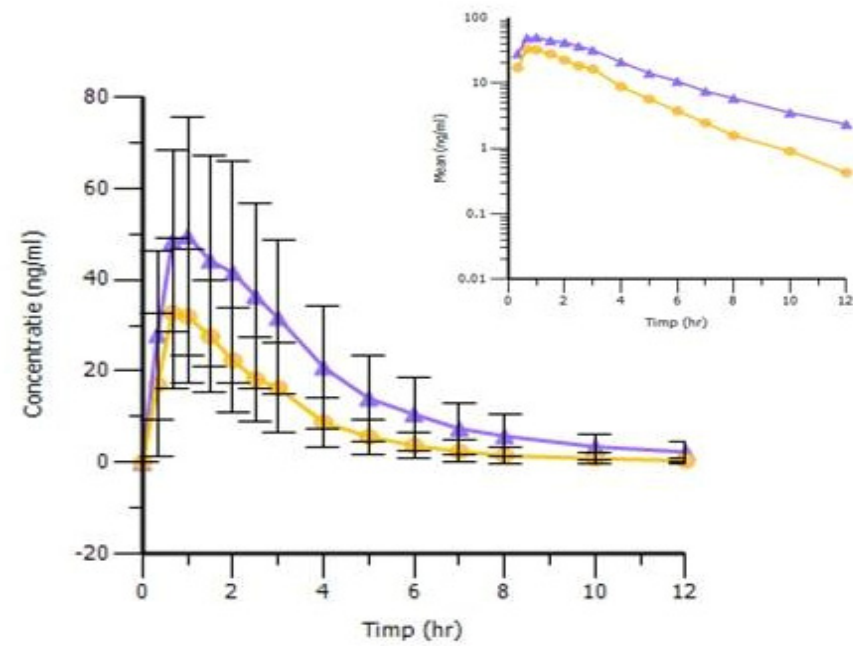

Figure 2. Mean \pm SD plasma concentration-time curves of zolpidem

(single dose $5 \mathrm{mg}$ p.o.) administered alone $(\mathbf{\Lambda})$ or in combination with phenytoin

(150 mg, p.o.), after pre-treatment with phenytoin during 6 days $(\bullet), n=20$. In the insert a logarithmic scale is employed. 
DANA MARIA MUNTEAN, MARIA ADRIANA NEAG, DANIELA PETRUTA PRIMEJDIE, ADINA POPA, MARCELA ACHIM, LAURIAN VLASE

The mean values of pharmacokinetic parameters [16] of zolpidem administered alone, or in combination with phenytoin, as well as statistical ANOVA test results are given in Table 1.

Table1. Pharmacokinetic parameters of zolpidem administered alone or after treatment with phenytoin and the result of statistical ANOVA test (in form of $p$ values) used for comparison

\begin{tabular}{|c|c|c|c|}
\hline $\begin{array}{c}\text { Parameter } \\
( \pm \mathrm{SD})\end{array}$ & $\begin{array}{c}\text { Zolpidem } \\
\text { (Reference) }\end{array}$ & $\begin{array}{c}\text { Zolpidem + Phenytoin } \\
\text { (Test) }\end{array}$ & $\boldsymbol{p}^{*}$ value \\
\hline $\mathrm{C}_{\max }(\mathrm{ng} / \mathrm{mL})$ & $56.6 \pm 25.4$ & $36.0 \pm 14.6$ & $0.0005, \mathrm{~S}$ \\
\hline $\mathrm{t}_{\max }(\mathrm{hr})$ & $0.8 \pm 0.3$ & $0.8 \pm 0.4$ & $0.816, \mathrm{NS}$ \\
\hline $\mathrm{AUC}_{0-\infty}(\mathrm{ng} \cdot \mathrm{hr} / \mathrm{mL})$ & $211.5 \pm 119.6$ & $103.5 \pm 54.0$ & $0.0009, \mathrm{~S}$ \\
\hline $\mathrm{k}_{\mathrm{el}}(1 / \mathrm{hr})$ & $0.3 \pm 0.1$ & $0.4 \pm 0.1$ & $0.0411, \mathrm{~S}$ \\
\hline $\mathrm{t} / \mathrm{hr})$ & $2.5 \pm 0.9$ & $1.7 \pm 0.5$ & $0.0411, \mathrm{~S}$ \\
\hline $\mathrm{Cl}(\mathrm{mL} / \mathrm{hr})$ & $34290.4 \pm 27007.8$ & $61587.0 \pm 32764.4$ & $0.0009, \mathrm{~S}$ \\
\hline
\end{tabular}

* Statistically significant (symbolized S) when $p<0.05$; NS stands for non-significant

The present study showed that phenytoin had an important influence on the pharmacokinetics of zolpidem. A systemic metabolic drug-drug interaction was observed since the half-life of zolpidem was significantly decreased. This may due to the phenytoin's enzymatic induction of hepatic CYP3A4, CYP2C9 and CYP2C19. However, zolpidem's exposure $\left(C_{\max }\right.$ and $\left.A \cup C_{0-\infty}\right)$ was also significantly reduced by the pre-treatment/co-administration of phenytoin; this indicated a pre-systemic metabolic drug-drug interaction. The metabolism of zolpidem occurs predominantly through CYP3A4, CYP2C9 and CYP1A2 isoenzymes [10]; the CYP3A4 isoenzymes are the predominant ones at both the intestinal and hepatic levels, whereas the others are mainly present in the liver [23]. Phenytoin induces CYP3A4 at both the intestinal and hepatic level and consequently, the first-pass metabolism of zolpidem was increased [24]. As an effect of a pre-systemic pharmacokinetic drug-drug interaction, the exposure of zolpidem was significantly lowered.

This study offers a first perspective on the magnitude of the pharmacokinetic interaction between zolpidem and phenytoin administered to human subjects. Results may contribute to the establishment of effective dosing intervals or treatment regimens [25]. For example, insomnia is common in epilepsy and it was associated with short term poor seizure control and worse quality of life [26]. Sleep disorders are frequently associated with various neurologic conditions, including persistent neuropathic pain, for which phenytoin has an on-label indication. Therefore, such clinical settings might require the coprescription of phenytoin and zolpidem, in spite of their additive central nervous system depressant effect [27]. If such association is implemented, then the pharmacokinetic interaction should be taken into consideration in situations when a long-term phenytoin treatment is to be stopped. This scenario favors an 
increased risk for zolpidem overdose and side-effects, including daytime residual effects on cognitive and psychomotor performance [28]. On the other hand, the initiation of phenytoin in a patient exposed to a chronic therapy with zolpidem could trigger signs and symptoms of zolpidem withdrawal that can complicate the patient's clinical state [29].

We identified a single previous investigation analyzing the simultaneous use of zolpidem and phenytoin, which focused on a pharmacodynamic aspect of their interaction, suggesting an increased antiepileptic effect. It was demonstrated on Wistar rats; it was supposed to be mediated through hyper-polarization, secondary to membrane stabilizing activity through sodium channel blockade by phenytoin and to reinforced GABA mimetic action of zolpidem [30].

Therefore, these results bring new information referring to the pharmacokinetic interactions previously confirmed for both zolpidem and phenytoin [31-34].

\section{CONCLUSIONS}

Administration of multiple-dose phenytoin influenced zolpidem pharmacokinetics in healthy volunteers, decreasing its exposure due to enzymatic induction.

\section{EXPERIMENTAL SECTION}

\section{Subjects}

Twenty healthy adult male subjects aged 22 to 30 years were eligible for the study. The health status of each volunteer was assessed using the individual medical history, the results of physical examinations, vital signs, electrocardiogram evaluation, and laboratory test results. Exclusion criteria included: use of any non-prescription drug products within 14 days before initiation of the study; use of prescription drugs during the 30-day period before the start of the study; use of other investigational drugs within 60 days before initiation of the study; donations or transfusions of blood or blood products during the 60-day period before the start of the study; smoking of more than 10 cigarettes per day; history of drug abuse; sitting systolic blood pressure $\geq 140 \mathrm{~mm} \mathrm{Hg}$ or $\leq 90 \mathrm{~mm} \mathrm{Hg}$; or sitting diastolic blood pressure $\geq 90 \mathrm{~mm} \mathrm{Hg}$ or $\leq 50 \mathrm{~mm} \mathrm{Hg}$.

The study was conducted according to the principles of Declaration of Helsinki (1964) and its amendments (Tokyo 1975, Venice 1983, Hong Kong 1989) and Good Clinical Practice (GCP) rules. The clinical protocol was reviewed and approved by the Ethics Committee of the University of Medicine and Pharmacy "Iuliu Hatieganu", Cluj-Napoca, Romania. 
DANA MARIA MUNTEAN, MARIA ADRIANA NEAG, DANIELA PETRUTA PRIMEJDIE, ADINA POPA, MARCELA ACHIM, LAURIAN VLASE

\section{Study design and blood sampling}

The study design was one sequence cross-over and consisted in 2 periods: Period 1 (Reference), when each volunteer received a single dose of 5 $\mathrm{mg}$ zolpidem and Period 2 (Test), when each volunteer received a single dose of $5 \mathrm{mg}$ zolpidem and $150 \mathrm{mg}$ phenytoin. Between the two periods, the subjects were treated for 6 days with a single daily dose of $150 \mathrm{mg}$ phenytoin. All drugs were administered in the morning, in fasted state. The pharmaceutical products used were Sanval $(5 \mathrm{mg}$ coated tablets, LEK Pharmaceuticals D.D. - Slovenia) and Fenitoin ${ }^{\circledR}$ (phenytoin 100 mg scored tablets, Gedeon Richter Romania, Târgu-Mures, Romania). Serial blood samples (5 mL each) were collected from an indwelling IV catheter immediately before drug administration and at $0.5,1$, $1,5,2,2,5,3,4,5,6,7,8,10$ and 12 hours, respectively, after drug administration and for each treatment period. Blood samples were collected in heparinized tubes. Samples were centrifuged at $1800 \mathrm{~g}$ for $5 \mathrm{~min}$ and harvested plasma samples were stored at $-20^{\circ} \mathrm{C}$ until analysis.

\section{Analysis of plasma samples}

Zolpidem plasma concentrations were determined by a validated high throughput liquid chromatography-mass spectrometry method, by using citalopram as an internal standard. The HPLC system was an Agilent 1100 series (Agilent Technologies, USA) and was coupled with a Brucker lon Trap SL (Brucker Daltonics $\mathrm{GmbH}$, Germany). A Zorbax SB-C18 chromatographic column (100 mm x $3.0 \mathrm{~mm}$ i.d., $3.5 \mu \mathrm{m}$ ) (Agilent Technologies) was used. The mobile phase consisted of 62:38 (V/V) $1 \mathrm{mM}$ ammonium acetate in water:methanol. The flow rate was $1 \mathrm{~mL} / \mathrm{min}$ and the thermostat temperature set at $45^{\circ} \mathrm{C}$. The mass spectrometry detection was in multiple reaction monitoring mode (MRM), positive ions, using an electrospray ionization source. The ion transitions monitored were $\mathrm{m} / \mathrm{z} 308 \rightarrow(\mathrm{m} / \mathrm{z} 235+\mathrm{m} / \mathrm{z} 263)$ for zolpidem and $\mathrm{m} / \mathrm{z} 325 \rightarrow \mathrm{m} / \mathrm{z}$ 262 for the internal standard.

$0.6 \mathrm{~mL}$ of methanol (containing internal standard, $10 \mathrm{ng} / \mathrm{mL}$ ) were added to $0.2 \mathrm{~mL}$ of plasma in an Eppendorf tube. The tube was vortex-mixed for 20 seconds, and then centrifuged for $3 \mathrm{~min}$ at $12000 \mathrm{rpm}$. The supernatant was transferred to an autosampler vial and $1 \mu \mathrm{L}$ was injected into the LC/MS system. The calibration curve of zolpidem was linear at a concentration range of 2 - $208 \mathrm{ng} / \mathrm{mL}$ plasma, with a correlation coefficient of 0.996 . At quantification limit $(2 \mathrm{ng} / \mathrm{mL}$ ), accuracy and precision were: $3.9 \%$ and $7.4 \%$ (intra-day) and $9.2 \%$ and $10.5 \%$ (inter-day), respectively.

\section{Pharmacokinetic analysis}

Non-compartmental pharmacokinetic analysis was employed to estimate the pharmacokinetic parameters of zolpidem when administered alone or in combination with phenytoin. The maximum plasma concentration 
PHARMACOKINETIC PROOFS ON INTERACTION BETWEEN ZOLPIDEM AND PHENYTOIN: A TWO-TREATMENT, TWO-PERIOD STUDY IN HEALTHY MALE SUBJECTS

$\left(\mathrm{C}_{\max }, \mathrm{ng} / \mathrm{mL}\right)$ and the time to reach the peak concentration $\left(\mathrm{t}_{\max }, \mathrm{hr}\right)$ were obtained directly by visual inspection of each subject's plasma concentrationtime profile. The area under the concentration-time curve $\left(\mathrm{AUC}_{0-\mathrm{t}}\right)$ was estimated by integration. The area was extrapolated to infinity $\left(A \cup C_{0-\infty}\right)$ by addition of $C_{t} / k_{\text {el }}$ to $A_{U C} C_{0-t}(t$ in the index indicates the time resolved value). The elimination rate constant $\mathrm{k}_{\mathrm{el}}$ was estimated by the least-square regression of plasma concentration-time data points lying in the terminal region, by using a semilogarithmic dependence that corresponds to first-order kinetics. The half-life $\left(\mathrm{t}_{1 / 2}\right)$ was calculated as the ratio $0.693 / \mathrm{kel}_{\mathrm{el}}$. The pharmacokinetic analysis was performed using Kinetica 4.2 (Thermo Labsystems, U.S.A.).

\section{Statistical analysis}

Analysis of variance (ANOVA testing) was used to compare the calculated pharmacokinetic parameters of zolpidem for the two periods, using general linear model procedures, in which sources of variation were subject and period. In order to identify possibly clinically significant differences in pharmacokinetic parameters, $90 \%$ confidence intervals of the test/reference period ratios for $\mathrm{C}_{\max }, \mathrm{AUC}_{0-\mathrm{t}}$ and $\mathrm{AUC}_{0-\infty}$ (log transformed) were determined by the Schuirmann's two one-sided $t$-test. The equivalence between zolpidem administered alone or in combination with phenytoin was inferred if the $90 \%$ confidence intervals for these pharmacokinetic parameters were within the range $0.8-1.25$. For $t_{\max }$, the equivalence range was expressed as untransformed data, and significance tested using the nonparametric Friedman test. All statistical analysis were performed using the Kinetica 4.2 software.

\section{ACKNOWLEDGMENTS}

This work was supported by a PNII-IDEI project, code 462, contract 229/2007 financed by CNCS Romania, for which the authors gratefully acknowledge.

\section{REFERENCES}

1. J. MacFarlane, C. M. Morin, J. Montplaisir, Clin Ther., 2014, 36(11), 1676.

2. Truven Health Analytics. Micromedex® 2.0. Zolpidem. DRUGDEX® Evaluations. Available at: http://www.micromedexsolutions.com/home/dispatch. Accessed February 2017.

3. E.L. Sutton, Med. Clin. N. Am., 2014, 98, 565.

4. A. Qaseem, D. Kansagara, M.A. Forciea, et al., Ann. Intern. Med., 2016, 165(2).

5. J.A. Generali, D.J. Cada, Hosp. Pharm., 2011, 46(2), 101.

6. R. Ciurleo, P. Bramanti, R.S. Calabrò, Drugs, 2013, 73(17), 1849.

7. M. Puthiyathu, H. Greenspan, W. Levitt, et al., Psychiatric Annals, 2013, 43(3), 96.

8. A.C. Fitzgerald, B.T. Wright, S.A. Heldt, Psychopharmacology, 2014, 231, 1865. 
DANA MARIA MUNTEAN, MARIA ADRIANA NEAG, DANIELA PETRUTA PRIMEJDIE, ADINA POPA, MARCELA ACHIM, LAURIAN VLASE

9. Ambien®- zolpidem tartrate tablet, film coated. Sanofi-Aventis U.S. LLC. Drug label information. US National Library of Medicine. DailyMed - official provider of FDA label information. Available at:

http://dailymed.nIm.nih.gov/dailymed/druglnfo.cfm?setid=c36cadf4-65a4-4466b409-c82020b42452. Accessed November 2015.

10. L.L. von Moltke, D.J. Greenblatt, B.W. Granda, et al., Br. J. Clin. Pharmacol., 1999, 48(1), 89.

11. M. Bialer, Epilepsia, 2012, 53(7), 26.

12. Agence nationale de sécurité du médicament et des produits de santé. Résumé des caractéristiques du produit Di-Hydan $\circledast 100 \mathrm{mg}$, comprimé sécable. Available at: http://base-donnees-publique.medicaments.gouv.fr/affichageDoc.php?specid= 66303560\&typedoc=R. Accessed February 2017.

13. H. Torbic, A.A. Forni, K.E. Anger, et al., Am. J. Health. Syst. Pharm., 2013, 70(9), 759.

14. R. Bauer, M. Ortler, M. Seiz-Rosenhagen, et al., Neurosurg. Rev., 2014, 37(3), 381.

15. G.L. Birbeck, J.A. French, E Perucca, et al., Epilepsia, 2012, 53(1), 207.

16. J.O. McNamara, Pharmacotherapy of the Epilepsies. In L. Brunton, J. Lazo, K. Parker, Eds. Goodman \& Gilman's The pharmacological basis of therapeutics. 11th ed. New York: The McGraw-Hill Companies, Inc; 2005. Chapter 19.

17. Truven Health Analytics. Micromedex® 2.0. Phenytoin. DRUGDEX® Evaluations. Available at: http://www.micromedexsolutions.com/home/dispatch. Accessed February 2017.

18. J. Tanaka, H. Kasai, K. Shimizu, et al., Eur. J. Clin. Pharmacol., 2013, 69(3), 489.

19. P.N. Patsalos, D.J. Berry, B.F. Bourgeois, et al., Epilepsia, 2008, 49(7), 1239.

20. R.J. Porter, B.S. Meldrum, Antiseizure Drugs. In B.G. Katzung, S.B. Masters, A.J.Trevor Basic \& Clinical Pharmacology, $12^{\text {th }}$ ed. New York: The McGraw-Hill Companies, Inc; 2012. Chapter 24.

21. C. Hiemke, P. Baumann, N. Bergemann, et al., Pharmacopsychiatry, 2011, 44(6), 195.

22. M.D. Krasowski, L.E. Penrod, BMC Medical Informatics and Decision Making, 2012 , $12,7$.

23. A. Galetin, M. Gertz, J.B.Houston, Drug. Metab. Pharmacokinet., 2010, 25(1), 28.

24. I. Cascorbi, Dtsch. Arztebl. Int., 2012, 109(33-34), 546.

25. T. Roehrs, T. Roth, Neurotherapeutics, 2012, 9, 728.

26. M. Quigg, S. Gharai, J. Ruland, et al., Epilepsy Res., 2016, 122, 91.

27. M.D. Cheatle, S. Foster, A. Pinkett, et al., Anesthesiology Clin. 2016, 34, 379.

28. N. Gunja, J. Med. Toxicol., 2013, 9, 155.

29. M.J. Brodie, S. Mintzer, A.M. Pack, et al., Epilepsia, 2013, 54(1), 11.

30. C. Gurudeva, P.A. Patil, S.V. Hiremath, et al., Pharmacologyonline, 2008, 3, 978.

31. K. Villikka, K.T. Kivistö, H. Luurila, et al., Clin Pharmacol. Ther., 1997, 62(6), 629.

32. Y. Hojo, M. Echizenya, T. Ohkubo, et al., J Clin Pharm Ther., 2011, 36(6), 711.

33. J.F. Okulicz, G.A. Grandits, J.A. French, et al., Epilepsy Res., 2013, 103(2-3), 245.

34. W.K. Kennedy, M.W. Jann, E.C. Kutscher, CNS Drugs, 2013, 27(12), 1021. 